\section{Obesity and reproduction}

\author{
Jane E Ramsay, Ian Greer, Naveed Sattar
}

The effect of adiposity is manifest in nearly every aspect of female reproductive life, whether as a metabolic or reproductive complication or as a technical problem affecting clinical issues such as ultrasonography or surgery. Indeed, obesity is present in $35 \%$ of maternal deaths in the United Kingdom.

Such concerns are particularly important given recent evidence of a doubling in the prevalence of obesity in young women attending for antenatal care in maternity hospitals in the UK (in some places, almost one in five are now obese). In the United States, where obesity rates are generally even higher, the American College of Obstetricians and Gynecologists has issued guidance on the impact of obesity on pregnancy.

\section{Gynaecological concerns}

Strong evidence shows that insulin resistance is an integral part of polycystic ovarian syndrome, especially in obese women. In most women with the syndrome, hyperinsulinaemia-driven or revealed by excess weight gain-promotes ovarian androgen secretion and abnormal follicular development, leading to dysfunctional ovarian and menstrual activity.

Androgens are carried in the circulation bound to sex hormone binding globulin (SHBG). Conditions of high androgen and insulin concentrations are associated with lower levels of SHBG, resulting in high free androgen activity. Thus, clinical manifestations of polycystic ovarian syndrome are associated with androgen activity and include hirsutism, acne, and alopecia, as well as oligomenorrhoea and ovulation failure.

Medications that contain oestrogen (such as the combined contraceptive pill) or ovulation induction drugs (resulting in high levels of endogenous oestrogen) may be associated with an increased risk of venous thromboembolism in obese women. The combined effect of obesity and the combined contraceptive pill results in a 10-fold increased risk of venous

thromboembolism in women with a body mass index of $>25$ (body mass index is calculated by dividing weight in kilograms by height in metres squared). Therefore routine management options for the gynaecological associations of obesity are to a certain extent contraindicated.

The potential for a significantly complicated pregnancy in obese women is high and therefore in a woman with impaired fertility owing to obesity, the ethical concern is whether to start medical management before a serious attempt at weight loss through diet and exercise.

\section{Obstetric concerns}

Subfertility treatment and early pregnancy outcome Some data suggest that the most clinically useful predictors of poor outcome from gonadotrophin ovulation induction in women with normogonadotrophic anovulatory infertility are obesity and insulin resistance. Also, an obese woman is three times more likely to miscarry. This association further emphasises the importance of encouraging weight loss to maximise the chance of a successful pregnancy before starting to treat anovulatory subfertility.

\begin{tabular}{lll}
\hline Potential effects of adiposity before and during pregnancy \\
& Medical complications & $\begin{array}{l}\text { Technical } \\
\text { complications }\end{array}$ \\
\hline $\begin{array}{l}\text { Prepregnancy } \\
\text { pregnancy }\end{array}$ & Menstrual disorders, infertility & \\
\hline Antenatal & $\begin{array}{l}\text { Pregnancy induced hypertension, } \\
\text { pre-eclampsia, gestational diabetes, } \\
\text { venous thromboembolism }\end{array}$ & $\begin{array}{l}\text { Difficult } \\
\text { ultrasound } \\
\text { examination }\end{array}$ \\
\hline Intrapartum & $\begin{array}{l}\text { Induction of labour, shoulder } \\
\text { dystocia, caesarean section }\end{array}$ & $\begin{array}{l}\text { Operative or } \\
\text { anaesthetic } \\
\text { problems }\end{array}$ \\
\hline Postpartum & $\begin{array}{l}\text { Haemorrhage, infection, venous } \\
\text { thromboembolism }\end{array}$ \\
\hline Fetal & $\begin{array}{l}\text { Macrosomia, fetal distress, } \\
\text { perinatal morbidity/mortality }\end{array}$ & Birth injury \\
\hline
\end{tabular}

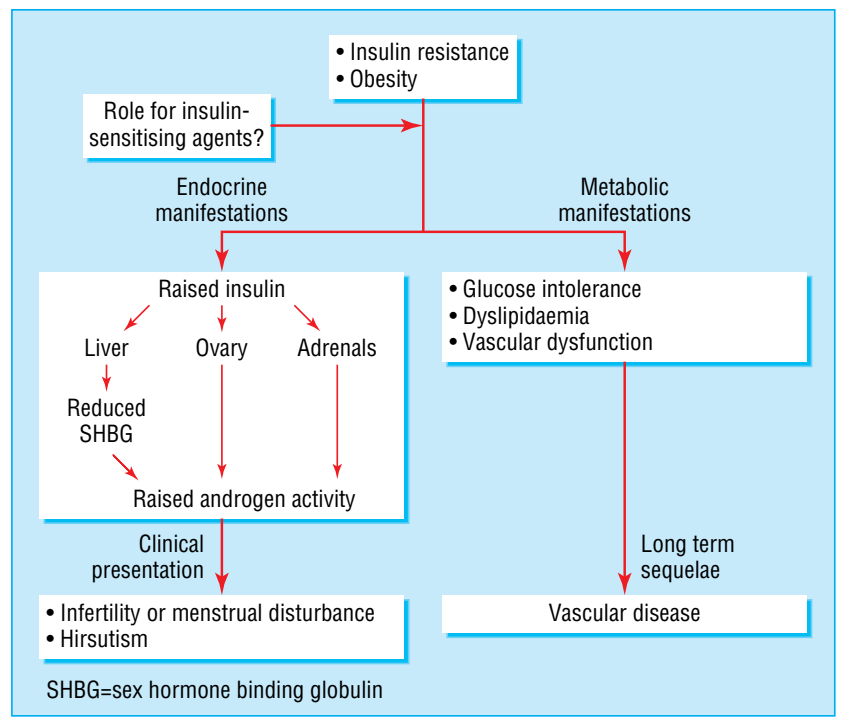

Endocrine and clinical effects of obesity and insulin resistance in women. Adapted from Harborne et al. Lancet 2003;361:1894-901

Obese women are about three times as likely as non-obese women to have an infant with either spina bifida or an omphalocele and about twice as likely to have a baby with either a heart defect or multiple anomalies

Although folic acid supplementation before conception is recommended for all women, the increased risk of neural tube defects in obese women has persisted in populations where flour has been fortified with folic acid 
Risks of pregnancy complications associated with obesity, according to five studies. Risks expressed as odds ratio (95\% confidence interval) or percentage (US cohort study)

Study (by complication)

Risk of complication

Miscarriage

Meta-analysis (13 studies) in women with normogonadotrophic $\quad$ Rate of spontaneous miscarriage 3.05 (1.4 to 6.4)

anovulatory infertility examined patient predictors for outcome

of ovulation induction with gonadotrophins: obese $v$ non-obese

women

Fetal anomalies

Case-control study of major birth defects in about 40000

births/year in 1993-97

Spina bifida 3.5 (1.2 to 10.3), omphalocele 3.3 (1.0 to 10.3), heart defects 2.0

All complications

UK study of 287213 singleton pregnancies (62\% normal

weight, $28 \%$ overweight, $11 \%$ obese): obese versus normal weight (1.2 to 3.4$)$

US cohort study of 613 morbidly obese and 11313 non-obese women Gestational diabetes 3.6 (3.25 to 3.98$)$, pre-eclamptic toxaemia 2.14 (1.85 to $2.47)$, macrosomia 2.36 (2.23 to 2.50$)$, intrauterine death 1.4 (1.14 to 1.71$)$, induction of labour 1.70 (1.64 to 1.76 ), caesarean section 1.83 (1.74 to 1.93 ), postpartum haemorrhage 1.39 (1.32 to 1.46 ), genital infection 1.3 (1.1 to 1.6 ), urinary tract infection 1.4 (1.2 to 1.6$)$, wound infection 2.2 (2.0 to 2.6)

distress $12 \%$ ข $8 \%$, caesarean section $31 \%$ v $15 \%$, endometritis $9 \%$, $3 \%$

Case control study from Middle East of 159 obese women and Gestational diabetes mellitus 26 (9 to 73), pregnancy induced hypertension 300 women of normal weight, matched for age and parity

\section{Metabolic complications}

\section{Hypertension}

The risks of pregnancy induced hypertension or pre-eclampsia are significantly greater if the woman is overweight. Most published work suggests a twofold to threefold increase in risk of pre-eclampsia if early pregnancy body mass index $(>30)$ or waist circumference $(>88 \mathrm{~cm})$ is used as an indicator of obesity.

\section{Diabetes}

Maternal obesity is associated with a fourfold increase in risk of gestational diabetes. In the short term, appropriate management of gestational diabetes can reduce the incidence of fetal macrosomia and serious perinatal morbidity, although it can increase the need for intervention.

In the long term, women with gestational diabetes are much more likely (severalfold higher risk) than the general population to develop diabetes, and this risk may be greatest in obese women. Therefore, in obese women with gestational diabetes, the early postnatal visit is an ideal opportunity for lifestyle advice on weight loss and exercise. Such women should be regularly screened for type 2 diabetes.

\section{Venous thromboembolism}

Venous thromboembolism is the leading cause of maternal death in the UK. Pregnancy is a prothrombotic state, with increases in coagulation factors and a decrease in natural anticoagulants along with inhibition of fibrinolysis.

Other factors likely to be important in the aetiology of venous thromboembolism associated with pregnancy are obesity, advanced maternal age, high parity, operative delivery, and pre-eclampsia, with these last four also being associated with maternal obesity.

\section{Technical concerns}

Ultrasonography in morbidly obese patients can be challenging. Adipose tissue attenuates the ultrasound signal by absorption of the associated energy. Therefore a high frequency, higher resolution signal would be more significantly absorbed at a lesser depth, requiring sacrifice of image quality for depth of field.

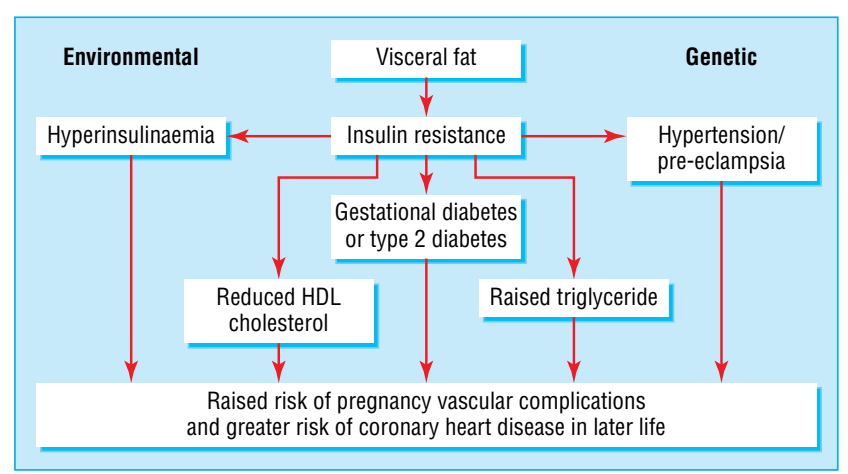

Adiposity, insulin resistance, and clinical effects in non-pregnant and pregnant women. A central body fat distribution can be influenced by both genetic and environmental factors. However, this phenotype is believed to be inextricably linked with the "metabolic syndrome." These factors are reflected in pre-eclampsia and gestational diabetes

Obesity seems to treble the risk of thrombosis, with greater likelihood of progression to pulmonary embolism, and obese individuals have higher levels of factor VIII and factor IX, but not of fibrinogen

Potential technical problems in managing obese mothers

- Difficult to use ultrasonography accurately to date pregnancy or detect fetal anomalies

- Large cuffs required to measure blood pressure accurately

- Woman may have reduced awareness of fetal movements

- External electronic fetal monitoring can be problematical

- Surgery is technically difficult, requiring more assistants or larger operating table

- Regional and general anaesthesia can be challenging

- Wound care can be compromised in the event of caesarean section 
A study of routine ultrasound screening in pregnant women with diabetes found that major congenital anomalies were almost six times as common in these women as in controls. The detection rate was significantly lower, however, for the diabetic women than for the controls $(30 \% v 73 \%$; $\mathrm{P}<0.01)$, and this was related to the higher incidence of obesity. This is important, as even in obese women without diabetes, epidemiological evidence consistently shows an increased rate of fetal anomalies.

Increased rates of intrapartum complications (such as failure to progress, shoulder dystocia, induction of labour, and emergency caesarean section) occur in many different populations of obese women. With the increased rate of caesarean sections in obese women, anaesthesia services need to be effective.

Many emergency sections for cephalopelvic disproportion or failure to progress occur at night-time, when many labour wards are staffed by the most junior staff, both obstetric and anaesthetic. So the least experienced staff may perform the most technically demanding and consequently high risk surgery and anaesthesia. In women who have had a previous section, the chance of successful vaginal delivery is $<15 \%$ for those who are very obese.

\section{Costs for maternal care}

As obesity can affect all aspects of maternal health, management of pregnancy in obese women is likely to be associated with a substantial increase in cost. One study has suggested that for obese women the cost of hospital antenatal care is about five times higher than the average.

\section{Fetal and neonatal concerns}

\section{Short term}

Maternal obesity is associated with an increased risk of fetal macrosomia, and data show that the incidence of birth weight of $\geq 4000 \mathrm{~g}$ is increased, regardless of whether the mother has diabetes. Macrosomia is a risk factor for a lower Apgar score at 1 minute and a lower umbilical arterial $\mathrm{pH}$ level, as well as for severe injuries to the baby (fractures and palsies). The overall morbidity for these babies is increased by about $8 \%$.

Data on risk of intrauterine growth restriction is conflicting. In general, intrauterine growth restriction does not seem to be associated with maternal obesity, which seems to protect against spontaneous preterm labour.

Despite this, the percentage of infants requiring admission to a neonatal intensive care unit seems to be significantly higher in children born to obese mothers, presumably secondary to the increased rates of antenatal complications and complications secondary to macrosomia. Obese women are also less likely to breast feed.

\section{Long term}

Research has suggested that impaired adult cardiovascular health may be programmed in utero by poor fetal nutrition, or by genetically determined attenuation of insulin mediated fetal growth, resulting in a small infant. Recent studies suggest, however, that obese mothers (even if they do not develop diabetes) may adversely programme their offspring in utero for greater obesity in later life (see Catalono, Further Reading box).

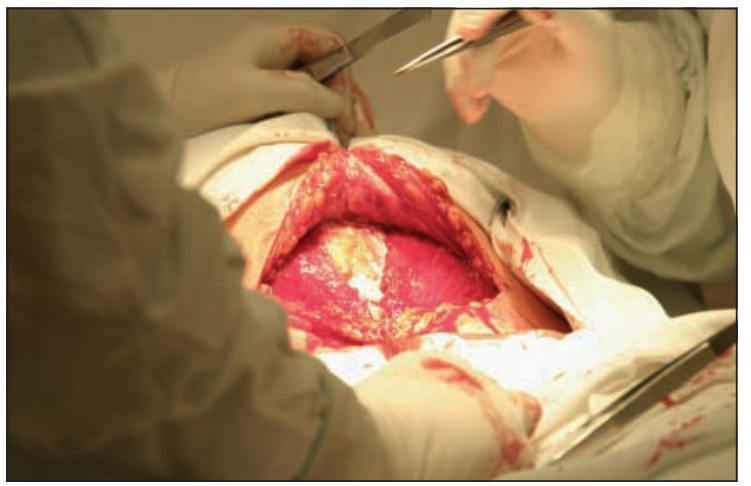

The mechanisms underlying the increased risks of intrapartum complications for obese women are elusive and do not simply reflect macrosomia. One hypothesis suggests increased soft tissue obstruction of labour, and dysfunctional uterine contractions may also be involved. In the postpartum period there is an increased rate of haemorrhage, genital tract infection, urinary tract infection, and wound infection

Why obesity can raise management costs of pregnancy

- Increased risk of admission to hospital for complications such as pre-eclampsia

- Increased use of ultrasonography and operator time for difficult anomaly scans and fetal assessment

- Increased risk of operative delivery and postpartum complications such as infection, haemorrhage, and venous thromboembolism

- Increased risk of neonatal admission

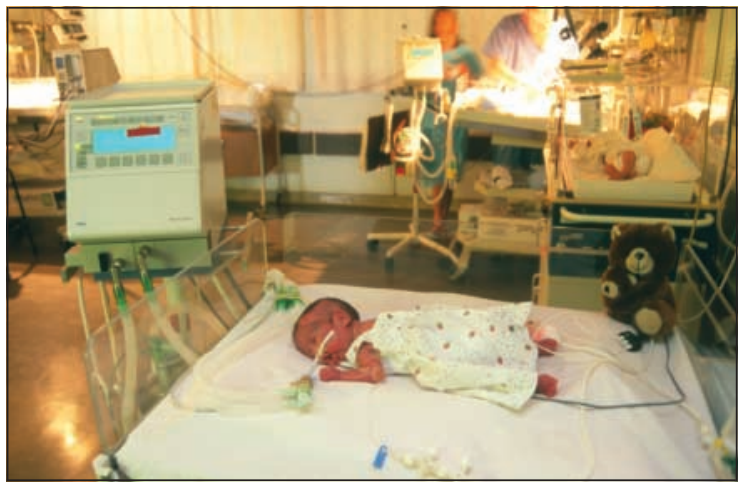

Maternal obesity is associated with an increased risk of neonates being admitted to a neonatal intensive care unit

Population based research shows a link between maternal obesity and cardiovascular disease in adult offspring. Also, higher adult rates of type 2 diabetes have been reported in offspring of mothers who were above average weight in pregnancy

Given that obesity and maternal insulin resistance is not only genetic but acquired, improvement of periconceptional maternal insulin sensitivity via activity or diet may affect not only a mother's health but also the future cardiovascular risk of her child. This hypothesis is speculative, however; further research is needed 


\section{Reproductive concerns in men}

The prevalence of some degree of erectile dysfunction in men aged $40-70$ years is thought to be about $50 \%$. Symptoms are increased in obese men, and the underlying pathophysiology is believed to be associated with the complications of the metabolic syndrome. In the past decade, erectile dysfunction has become a "hot topic" for developments in medical therapy. However, modifiable health behaviours, including weight loss and physical activity, are clearly associated with a reduced risk for erectile dysfunction.

\section{Intervention and outcome}

In obese women, we must consider modification of risk factors before, or early in, a pregnancy. Increased physical activity in women who are sedentary may result in a better pregnancy outcome for both mother and child. Preliminary data support this hypothesis, and some data also suggest beneficial effects of exercise in pregnancy on fetal development, showing significantly higher birth weights and faster mid-trimester growth rates. Larger trials are needed.

Exercise in pregnancy may also reduce pregnancy complications such as gestational diabetes and does not seem to be harmful, with no association with premature labour or poor Apgar scores. Data from the recent Cochrane meta-analysis on use of aspirin for prophylaxis of pre-eclampsia has suggested an overall reduction of risk of $15 \%$. However, when aspirin is targeted at women in high risk groups-for example, when multiple risk factors exist (such as obesity, age, family history) - it may have a more significant effect.

Finally, because of the considerable potential adverse impact of obesity on pregnancy outcome for both mother and baby, some researchers have even suggested that surgical treatment of obesity in young women may be warranted to prevent such complications.

Advice for achieving a healthy lifestyle should be actively disseminated by all health professionals at every opportunity, including the gynaecology clinic and antenatal and postnatal visits. Ideally, the risks of conceiving when obese should be disseminated through public health initiatives. Prepregnancy clinics could provide education on healthy diet and exercise regimens that could be followed before trying to conceive.

The photographs of a caesarean section and a neonatal intensive care unit are published with permission from Jacky Chapman/Photofusion and Garry Watson/SPL respectively.

Jane Ramsay is consultant obstetrician and gynaecologist at Ayrshire Maternity Unit, Kilmarnock, and Ian Greer is professor of obstetrics and gynaecology at the University of Glasgow.

The ABC of Obesity is edited by Naveed Sattar (nsattar@clinmed.gla.ac.uk), professor of metabolic medicine, and Mike Lean, professor of nutrition, University of Glasgow. The series will be published as a book by Blackwell Publishing in early 2007.
Suggestions for clinically managing pregnancy in obese women*

- Obese women should receive prepregnancy counselling (via clinics for subfertility, recurrent miscarriage, or diabetes; via obstetric prepregnancy clinics; or via a general practitioner) and folic acid supplements

- Encourage obese women to lose weight before conceiving, and to use contraception while aiming for target weight (fertility rises as body mass index decreases)

- Advise on the importance of healthy diet and exercise and the need to avoid excessive weight gain (refer to dietitian if necessary)

- Consider low dose aspirin in the presence of additional risk factors (obesity is associated with increased risk of pre-eclampsia); assess thrombosis risk and provide thromboprophylaxis if needed

- Recommend detailed anomaly scan and serum screening for congenital abnormality

- Consider glucose tolerance testing at 28 weeks

- Recommend anaesthetic review before delivery

- Plan delivery to allow optimum management by experienced obstetricians

- Consider prophylactic postpartum antibiotics if delivery is complicated

- Assess thrombosis risk postpartum and provide thromboprophylaxis if indicated

- Consider extended thromboprophylaxis after discharge

- Arrange postnatal review at six weeks to discuss any problems and potential for future intervention

*Best targeted at women with body mass index of $>35$ (rather than $>30$ ). Further data is needed for cost effectiveness of targeting all women with BM $>30$ as this would have major impact on clinical practice owing to high prevalence (near $20 \%$ ) of obesity in young women in some places, and thus needs a better evidence base

\section{Further reading and resources}

- Ramsay JE, Greer IA. Obesity in pregnancy. Fetal Matern Med Rev 2004;15:109-32.

- Kanagalingam MG, Forouhi NG, Greer IA, Sattar N. Changes in booking body mass index over a decade: retrospective analysis from a Glasgow maternity hospital. BJOG 2005;112:1431-3.

- Wong SF, Chan FY, Cincotta RB, Oats IJ, McIntyre HD. Routine ultrasound screening in diabetic pregnancies. Ultrasound Obstet Gynecol 2002;19:171-6.

- Galtier-Dereure F, Boegner C, Bringer J. Obesity and pregnancy: complications and cost. Am J Clin Nutr 2000;71:1242-8S.

- Callaway LK, Prins JB, Chang AM, McIntyre HD. The prevalence and impact of overweight and obesity in an Australian obstetric population. Med J Aust 2006;184:56-9.

- Catalano PM. Obesity and pregnancy-the propagation of a viscous cycle? J Clin Endocrinol Metab 2003;88:3505-6.

- American College of Obstetricians and Gynecologists' guidance on the impact of obesity on pregnancy. 2005. www.acog.org/ from_home/publications/press_releases/nr08-31-05-2.cfm.

Competing interests: For series editors' competing interests, see the first article in this series.

BMJ 2006;333:1159-62 